\title{
Non-communicable diseases and implications for medical practice in Australia: a framework for analysis
}

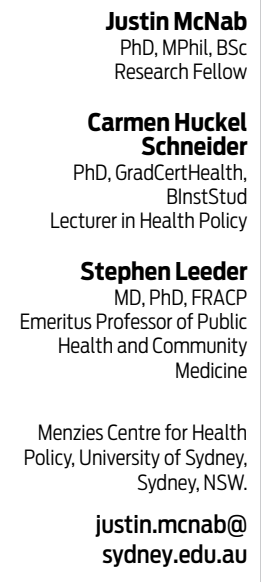

doi: 10.5694/mjal4.00161

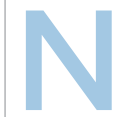

on-communicable diseases (NCDs) have taken over from communicable diseases as leading causes of mortality and morbidity. This is the result of several historical transitions, including: (i) the change in burden of disease from communicable to non-communicable (epidemiological transition); ${ }^{1}$ (ii) new patterns of fertility and ageing populations (demographic transition); and (iii) shifts in food availability and diet (nutritional transition). ${ }^{2}$ We know that the rate of deaths from communicable diseases in Australia began decreasing significantly from the late 19th century, culminating in low rates of all-cause mortality around 1945-1979 for men and 1960-1970 for women. ${ }^{3}$ During the 20th century, the rate of deaths from NCDs increased, with cardiovascular disease and cancer, in particular, becoming the leading causes of death among Australians. ${ }^{4}$

There has been considerable historical analysis of the underlying causes of this change, including how economic, social, cultural and political changes have influenced its extent and nature in different regions of the world. There has been far less historical analysis of how this transition has shaped medical practice.

Here, we lay out a framework for analysing changes to medical practice with respect to this transition. We highlight key historical features that indicate how each domain in our framework is critical for understanding how medical practice has changed for patients and health practitioners since the beginning of the 20th century. We propose the following four analysis domains aligned with key areas of change.

- Domain 1: changes in how disease is conceptualised, including how medical practitioners and patients have come to view sickness and the role of medicine and medical practitioners differently;

- Domain 2: changes in technology, its emergence, adoption and integration into standard care;

- Domain 3: changes in workforce, roles, and emerging areas of specialisation; and

- Domain 4: changes in the structure of health care, including models of care, and implications for health systems.

The purpose of the framework is to strengthen further inquiry and put forward propositions about how each of the four domains can aid historical analysis. Our aim is to encourage analysis that takes into account key features in each of the domains, thus enabling a more complete understanding of why, how and under what circumstances NCDs have had an effect on medical practice.

We use cancer, diabetes and cardiovascular disease as examples to illustrate possible starting points in each of the four domains.

\begin{abstract}
Summary
Non-communicable diseases (NCDs) have become leading causes of mortality and morbidity as part of historical epidemiological, demographic and nutritional transitions.

- There has been considerable historical analysis of the immediate and underlying causes of this change in the impacts of communicable diseases and NCDs, but far less historical analysis of how this transition has shaped medical practice.

We lay out a framework for future historical analysis by proposing four domains of inquiry into key areas of change: changes in the concept of disease; evolution of medical technology; changes in workforce, including variation in roles and emerging areas of specialisation; and changes in health care structures including models of care, government responses and transitioning health systems.

- Our aim is to encourage analysis that takes into account key features in each of the four domains, thus enabling a more complete understanding of why, how and under what circumstances NCDs have had an effect on medical practice.
\end{abstract}

\section{The four domains}

\section{Understanding how the concept of chronic disease has arisen and changed}

The first domain of the framework focuses on the changing conceptualisation of disease and how new ways of thinking influenced the way in which practitioners viewed their role. New concepts shaped demands on medical practice and changed the medical profession's ideas about what was important, possible and best care. ${ }^{5}$

In the late 1800s, NCDs such as cancer and cardiovascular disease were considered inevitable and a part of the natural process of ageing. These diseases were referred to variously as "degenerative", "disabling", "man-made", "chronic", "diseases of affluence" or, more recently, "non-communicable". The use of each term corresponds with different conceptualisations of diseases, their causes and the approach of medical practice to their treatment, control, management and prevention. For example, whereas in the 18th century the physician identified particular varieties of disease by characteristics and outward appearances, the role of the medical practitioner in the 19th and early 20th centuries became to detect the pathological process of disease within the body, which may or may not be outwardly visible. ${ }^{5}$ This allowed for a useful distinction where the abnormality could be identified as acute or persisting over the long term and so be understood as "chronic". A condition that develops or remains for months or years is less straightforward to treat and manage. ${ }^{2,6}$ This situation is further complicated 
because people with chronic illnesses also experience acute episodes or exacerbations of their conditions.

An historical analysis of change in conceptualisations of disease could also determine implications for the patient. For example, by the mid 20th century in the context of cancer, medical practitioners expected people to be aware not only of risks, but also of early signs of disease within their bodies, with an associated obligation to seek medical attention. ${ }^{7}$ In the latter part of the 20th century, disease prevention became an important theme, with the debate focusing on the extent to which lifestyle change could be the responsibility of individuals.

Changing conceptualisation also affected the way practitioners assessed and measured disease. By the 1950s, there was an increased focus on patients' capacity to function as a measure of the severity of disease. ${ }^{6}$ By the end of the 20 th century, the conceptualisation of cancer had been modified so that the emphasis was on "control" as well as cure. This prompted acceptance that some cancer was preventable, an increasing percentage treatable with long remissions (and in some cases cure), with the remainder managed through aiming for improved quality of life. ${ }^{8}$

Historical analysis of changes in how NCDs are conceptualised could further our understanding of the roles of medical practitioners, patient expectations of the medical profession and changing priorities for medical research, funding, training and practice.

\section{New technology for new disease}

The second domain of the framework seeks to understand technological changes that have shaped how medical practice has evolved throughout the 20th century and into the 21 st century. During this period, rapid technological advancement transformed all areas of personal and professional life, with medical practice a profound example. This second domain encourages historical analysis of technological advancements that influenced how medical practitioners were able to approach NCDs.

For example, the rising prominence of cardiovascular disease in the 20th century prompted innovative technological advances. An analysis could begin by examining how ideas from the physiology laboratory were applied to the study of the heart through the use of electrocardiography. Electrocardiography was introduced in Australia in 1912 and, as the knowledge base grew rapidly, characteristic electrocardiograph traces were associated with various aspects of the heart's function. This knowledge was then used to assist in diagnosis and treatment. Findings were disseminated in the Australian Medical Journal and the Medical Journal of Australia. ${ }^{7,9}$ Further technological developments were associated with major advances in cardiothoracic and vascular surgery from the mid 20th century. Imaging and scanning technologies, as well as devices such as stents, have had a major impact on diagnosis and treatment in the late 20th and early 21st centuries. An historical analysis of this continually developing field may elicit reasons whether, why or under what conditions this trend is set to continue.

A similar analysis could be conducted in the field of cancer. X-ray therapy for cancer had begun by the beginning of the 20th century. ${ }^{8}$ Along with surgery, radiotherapy soon came to dominate cancer treatment, with an associated research and funding response emerging from the mid 1930s. By the mid 20th century, hormone treatment, chemotherapy, radiotherapy and surgery were available, although there was controversy and confusion as to which combinations of treatment were the most effective. The rapid changes in technology and, thus, state-of-the-art care meant that medical practitioners were required to update their knowledge. As a result, training became a key issue. By the latter half of the 20th century, many medical practitioners were still not well informed about treatment options. ${ }^{10}$ An historical analysis of the connections between the availability of new technology, trained staff and better treatment options could shed light on how these factors may still be in play in today's health care systems.

Treatment and management of diabetes is another example where technology has played a major role. The use of insulin spread throughout Australia almost immediately, thanks to a close connection between clinicians here and overseas, and articles in the Medical Journal of Australia that informed and educated medical practitioners. ${ }^{11-14}$ The development and use of insulin along with first urine and then blood sugar testing is a complex and ongoing story. Changes in technology and the knowledge associated with it create fast-paced, often debated best-practice standards which, in the case of NCDs, are complicated by the chronic multimorbid nature of the conditions. Analysis of these changes can trace the history and effect of NCD-related technology through periods of uncertainty, innovation, adaptation and, finally, training and integration into standard practice.

\section{A changing workforce responds to NCDs}

The third domain focuses on the health workforce. This domain is critical to understanding the changing role of medical practitioners specialising in NCDs (including generalists and allied health practitioners) and the relationships among them and the health system as a whole.

Perhaps the most obvious change in the health workforce during the 20th century was the trend towards specialisation from around 1910 through to the 1960s, gathering pace in the postwar years. ${ }^{15}$ A good example of this in Australia can be found in the field of cardiology. ${ }^{16}$ New technologies, as discussed above, shaped the growth of various subspecialties from the mid 20th century continuing to the present. ${ }^{16-18}$

Specialisation also increased rapidly in the diagnosis and treatment of cancer. The various components of treatment required new levels of coordination between specialists, generalists and allied health professionals. Over the course of the century, working arrangements came to resemble team care. By the 1980s, general practitioners worked as facilitators of holistic care, with oncology nurses also part of the team and social workers working towards resolving the complex family and social problems (including access to social services) that are consequences of NCDs. ${ }^{8}$

A similar evolution in specialisation for physicians, nurses and allied health professionals, followed by the emergence of care coordination, is apparent in the management of diabetes, a potentially fruitful case study for historical analysis. Clinicians who specialised in the field were beginning to publish by the late 1800s in Australia, ${ }^{11}$ and diabetes clinics appeared in hospitals in major cities early in the 20 th century. Nurses played key roles in the treatment and 
management of diabetes from the early part of the 20th century, specialising as educators and performing many diagnostic tests. ${ }^{11}$ This corresponded with broader changes in nurses' roles, especially from the 1970s, largely in response to the new community health centres and the emerging idea of what would later be called nurse practitioner roles. From the 1990s, with an increased focus on management of NCDs, respiratory, cardiac and diabetes nurse specialists could be found in community health centres, and making home visits to care for patients with NCDs, often in partnership with general practices. ${ }^{19}$ Allied health professionals became a feature of the health workforce from the second half of the 20th century, with physiotherapists, podiatrists, dietitians, occupational therapists, psychologists and counsellors becoming established in multidisciplinary teams in hospital, private and community health settings. ${ }^{20}$

Tracing the connections between the growth of specialties, new technologies, and the relationship of both to how various health professionals work together provides a fuller picture of how care can best be delivered in complex health systems.

\section{Changing models of care and implications for health systems}

The fourth domain targets analysis of broader system changes and brings together implications from the other three domains. The health workforce in Australia responded to NCDs through conceptualising disease in new ways, increasing specialisation, changing roles and practice, and increasing the emphasis on prevention and coordinated management of disease. These changes had implications for patients, but also for health systems.

In the 20th century, Australia, like most other Western countries, established universal health care and health insurance coverage for its population. Along with it, system costs and (in)efficiency have come to dominate health policy debate. Poor coordination of care has been singled out as the largest contributor to the burden of NCDs from a health system point of view. ${ }^{21-25}$ Commonly proposed solutions involve greater integration of multiple services through coordination, flexibility and continuity, along with policy and health system changes, including information technology solutions to improve the management of chronic disease. 8,23

An historical analysis can trace the development of new models of care for patients with chronic disease. For example, in Australia, the concept of coordinating care for people with cancer emerged from the 1950s. Team approaches to care were accepted by the 1970s, with multidisciplinary teams being common in cancer treatment by the 1980 s. $^{8}$ The same was true for shared care, the exchange of patient data and sharing of skills and knowledge, by the 1990s. ${ }^{26}$ By the late 1990s, multidisciplinary teams were becoming established in emergency departments of hospitals, specifically as a response to an ageing, chronically ill population that required more visits to emergency departments but not necessarily inpatient care. ${ }^{20}$

Changes in policy accompanied these developments. In Australia, chronic disease-specific policies were formulated at the federal level in the 1980s and the 1990s, and more recently at state and territory levels. ${ }^{27}$ In some cases these had a direct influence on the delivery of treatment from medical practitioners, such as the inclusion in the Medicare
Benefits Schedule of items that allow general practitioners to receive remuneration for preparing management and team care arrangement plans. ${ }^{28}$

Other chronic disease policy developments included the national chronic disease strategy, which specifies four key priority areas for action: prevention across the continuum; early detection and treatment; integration and continuity of prevention and care; and self-management. ${ }^{29}$ This strategy sought to establish preventive medicine and coordination of care as major components for medical practitioners to pursue. Other similarly aligned policies include the national service improvement frameworks for asthma, diabetes, osteoarthritis, rheumatoid arthritis and osteoporosis, and heart, stroke and vascular disease. ${ }^{30-33}$ While not all national initiatives will directly affect medical practice, an historical analysis of their value as hortatory policy instruments that encourage giving priority to a particular action (rather than using direct incentives or regulation) can increase understanding of their impact on expectations for the medical profession and doctor-patient relationships. This makes a key contribution to this analysis domain and overall framework.

\section{Conclusions}

We have outlined a framework detailing four domains and their implications for medical practice in relation to the rise of NCDs in the 20th and early 21st centuries in Australia. New ways of conceptualising disease, new technologies, increasing specialisation, changing roles and practice, and an increasing focus on prevention and management have all played their roles in this ongoing and still unfolding story. We hope the discussion of each of these domains will prompt further in-depth historical analysis. This analysis could determine more specifically the connections between each of these domains and interactions within them, both in terms of changing roles and practices for practitioners and the implications of these changes for patients, their families and carers, and for the future provision of health care. This is necessary and indeed an immediate and pressing concern, given that the social and economic burden of chronic disease will increase in Australia over the coming decades.

Competing interests: Stephen Leeder is the Editor-in-Chief of the Medical Journal of Australia.

Provenance: Commissioned; externally peer reviewed.

1 Omran AR. The epidemiologic transition. A theory of the epidemiology of population change. Milbank Mem Fund Q 1971; 49: 509-538.

2 Lewis MJ, MacPherson KL. Health transitions and the double disease burden in Asian and Pacific countries: some introductory observations. In: Lewis MJ, MacPherson KL, editors. Health transitions and the double disease burden in Asia and the Pacific: histories of responses to non-communicable and communicable diseases. Abingdon, UK: Routledge, 2013: 1-14.

3 Lewis MJ, Leeder SR. Two health transitions in Australia: the Western and the Indigenous. In: Lewis MJ, MacPherson KL, editors. Health transitions and the double disease burden in Asia and the Pacific: histories of responses to non-communicable and communicable diseases. Abingdon, UK: Routledge, 2013: 15-46.

4 Australian Bureau of Statistics. Causes of death, Australia, 2012. Canberra: ABS, 2014. (ABS Cat. No. 3303.0.) http://www.abs.gov.au/ausstats/abs@. nsf/Lookup/3303.0main+features100012012 (accessed Jun 2014).

5 Lewis MJ. The people's health: public health in Australia 1950 to the present. Westport, Conn: Praeger, 2003: 43.

6 Armstrong D. Chronic illness: a revisionist account. Sociol Health Illn 2014; 36: $15-27$.

7 Silverberg MD. A brief account of the electro-cardiograph and its clinical application. Aust Med J 1912; 1: 488. 
8 Lewis MJ. Medicine and care of the dying: a modern history. Oxford: Oxford University Press, 2007.

9 Silverberg MD. Notes on cardiac irregularities. Med J Aust 1914; 1: 1-3.

10 Morgan GW. Development of radiation oncology services in Australia. Int J Radiat Oncol Biol Phys 1996; 36: 219-232.

11 Martin FIR. A history of diabetes in Australia. Melbourne: Miranova Publishers, 1998.

12 Wilkinson JF. Insulin in general practice. Med J Aust 1924; 1 Suppl 1: 44-47.

13 Sprent J. An introduction to the use of "insulin". Med J Aust 1923; 2: 384-386.

14 McWhae DM. Severe diabetes treated with insulin. Med J Aust 1923; 2: 544-545.

15 Roemer MI. Primary care and physician extenders in affluent countries. Int J Health Serv 1977; 7: 545-555.

16 Bauer GE. Four chapters in the history of Australian cardiology. Postgrad Med J 1970; 46: 229-236.

17 Mahar LJ. Interventional cardiology in Australia. J Interv Cardiol 1995; 8: 55-58.

18 Juneja MS, Walters DL. Interventional practices in Australia and New Zealand: current trends. Indian Heart J 2007; 59 (2 Suppl B): B20-B24

19 Eley DS, Patterson E, Young J, et al. Outcomes and opportunities: a nurseled model of chronic disease management in Australian general practice. Aust J Prim Health 2013; 19: 150-158.

20 Johnson L, Cusick A. Emergency departments: an emerging context of Australian allied health practice. J Allied Health 2009; 38: E29-E35.

21 Nolte E, McKee M. Caring for people with chronic conditions: a health system perspective. Maidenhead, UK: McGraw Hill Open University Press, 2008.

22 Curry N, Ham C. Clinical and service integration: the route to improved outcomes. London: The King's Fund, 2010.
23 Zwar N, Harris M, Griffiths R, et al. A systematic review of chronic disease management. Canberra: Australian Primary Health Care Research Institute, 2006.

24 van Raak A, Meijer E, Meijer A, Paulus A. Sustainable partnerships for integrated care: the role of decision making and its environment. Int $J$ Health Plann Manage 2005; 20: 159-180.

25 Blendon RJ, Schoen C, DesRoches C, et al. Common concerns amid diverse systems: health care experiences in five countries. Health Aff (Millwood) 2003; 22: 106-121.

26 Moorhead R. Sharing care between allied health professionals and general practitioners. Aust Fam Physician 1995; 24: 1985.

27 Jeon YH, Jowsey T, Yen L, et al. Achieving a balanced life in the face of chronic illness. Aust J Prim Health 2010; 16: 66-74.

28 Newland J, Zwar N. General practice and the management of chronic conditions - where to now? Aust Fam Physician 2006; 35: 16-19.

29 National Health Priority Action Council. National Chronic Disease Strategy. Canberra: Australian Government Department of Health and Ageing, 2006.

30 National Health Priority Action Council. National service improvement framework for asthma. Canberra: Australian Government Department of Health and Ageing, 2006.

31 National Health Priority Action Council. National service improvement framework for diabetes. Canberra: Australian Government Department of Health and Ageing, 2006.

32 National Health Priority Action Council. National service improvement framework for osteoarthritis, rheumatoid arthritis and osteoporosis. Canberra: Australian Government Department of Health and Ageing, 2006.

33 National Health Priority Action Council. National service improvement framework for heart, stroke and vascular disease. Canberra: Australian Government Department of Health and Ageing, 2006. 